\title{
Loss of Resting Interhemispheric Functional Connectivity after Complete Section of the Corpus Callosum
}

\author{
James M. Johnston, ${ }^{1}$ S. Neil Vaishnavi, ${ }^{2}$ Matthew D. Smyth, ${ }^{1}$ Dongyang Zhang, ${ }^{2}$ Biyu J. He, ${ }^{2}$ John M. Zempel, ${ }^{3}$ \\ Joshua S. Shimony, ${ }^{2}$ Abraham Z. Snyder, ${ }^{2,3}$ Marcus E. Raichle ${ }^{2,3,4,5}$ \\ ${ }^{1}$ Departments of Neurosurgery, ${ }^{2}$ Radiology, ${ }^{3}$ Neurology, ${ }^{4}$ Neurobiology, and ${ }^{5}$ Biomedical Engineering, Washington University, St. Louis, Missouri 63110
}

\begin{abstract}
Slow $(<0.1 \mathrm{~Hz})$, spontaneous fluctuations in the functional magnetic resonance imaging blood oxygen level-dependent (BOLD) signal have been shown to exhibit phase coherence within functionally related areas of the brain. Surprisingly, this phenomenon appears to transcend levels of consciousness. The genesis of coherent BOLD fluctuations remains to be fully explained. We present a resting state functional connectivity study of a 6-year-old child with a radiologically normal brain imaged both before and after complete section of the corpus callosum for the treatment of intractable epilepsy. Postoperatively, there was a striking loss of interhemispheric BOLD correlations with preserved intrahemispheric correlations. These unique data provide important insights into the relationship between connectional anatomy and functional organization of the human brain. Such observations have the potential to increase our understanding of large-scale brain systems in health and disease as well as improve the treatment of neurologic disorders.
\end{abstract}

Key words: synchrony; functional connectivity; corpus callosotomy; epilepsy; fMRI; resting state

\section{Introduction}

Since the introduction of electroencephalography (EEG) by Berger (1929), the presence of widespread, spontaneous, ongoing activity in the brain has been universally appreciated. Although the frequency content and rough spatial topography of spontaneous EEG activity have been well characterized, the relationship of this activity to underlying brain systems remains poorly understood. Knowledge of the relationships between brain structure and function greatly increased after the introduction of functional neuroimaging, first with PET and then with functional magnetic resonance imaging (fMRI) (Raichle and Posner, 1994). These techniques have been extensively used together with carefully designed task paradigms to map the representation of sensory, motor and cognitive processes within the brain. More recently, it has been recognized that slow $(<0.1 \mathrm{~Hz})$ spontaneous fluctuations in the BOLD signal show phase correlation within widely distributed functional networks even in the absence of externally imposed tasks, i.e., at "rest" (Biswal et al., 1995; Fox and Raichle, 2007). Because of the striking spatial organization of this activity, it is reasonable to posit that anatomical connectivity provides an important constraint. Data on this point, however, are limited (Vincent et al., 2007).

To explore this issue, we took advantage of a unique clinical opportunity to study patterns of spontaneous fMRI BOLD activity in a single individual with intractable epilepsy both before and

Received Feb. 7, 2008; revised May 7, 2008; accepted May 13, 2008.

This work was supported in part by National Institutes of Health Grant NS06833. We thank Damien Fair, Bradley Schlaggar, and Steven Petersen for their helpful discussions regarding the role of development in our findings.

Correspondence should be addressed to James M. Johnston, Department of Neurosurgery, Washington University School of Medicine, Campus Box 8225, 4525 Scott Avenue, East Building, St. Louis, M0 63110. E-mail: johnstonj@nsurg.wustl.edu.

DOI:10.1523/JNEUROSCI.0573-08.2008

Copyright $\odot 2008$ Society for Neuroscience $\quad 0270-6474 / 08 / 286453-06 \$ 15.00 / 0$ after complete section of the corpus callosum for the treatment of intractable epilepsy. We were able to directly test whether disruption of a major anatomical pathway affected the correlation structure of spontaneous BOLD signal fluctuations.

\section{Materials and Methods}

Clinical details. The patient was a 6-year-old male with a four year history of idiopathic, intractable myoclonic and atonic seizures ( $\sim 75$ per day). Extensive workup revealed no history of CNS infection, systemic disease, or congenital/chromosomal abnormality. Neurologically, the patient was developmentally delayed but without focal deficits, nonverbal but interactive. Twenty-four hour video electroencephalogram evaluation showed generalized spike-wave activity without a localized seizure focus.

Preoperative structural MR images demonstrated normal anatomy with normal corpus callosum development and no evidence of cortical dysplasia or ischemic insult. The child underwent a single-stage complete transection of the entire corpus callosum, including the splenium and dorsal hippocampal commissure. The anterior and posterior commissures were left intact. There were no perioperative complications. Postoperative MRI confirmed complete transection and no evidence of hemorrhage, edema, or infarct. The patient was discharged on postoperative day 5 at his neurological baseline. Since discharge, the patient has done well with significantly reduced atonic seizure frequency and improvement in both social behavior and language function.

Data acquisition. Imaging was performed on a 3T Siemens Trio system. Functional data were acquired using a gradient echo, echo-planar sequence sensitive to BOLD contrast (echo time, $25 \mathrm{~ms}$; flip angle $90^{\circ} ; 4 \times$ $4 \times 5 \mathrm{~mm}$ voxels; field of view, 256; repetition time, $2.08 \mathrm{~s}$; bandwidth, $2605 \mathrm{~Hz}$ ). Three runs of 200 frames were completed. Whole-brain coverage was obtained with 32 contiguous slices. Structural data included a high-resolution, axial T1-weighted magnetization-prepared gradientecho (MP-RAGE) scan and a high-resolution T2-weighted fast spin echo scan for definitive atlas transformation. Because of this child's age and agitation, he was imaged under propofol general anesthesia per institutional protocol both preoperatively and postoperatively. BOLD fMRI was acquired at the time of these clinical scans. The child's mother pro- 
vided informed consent following guidelines approved by the Human Studies Committee of Washington University.

Preprocessing of functional data. Preprocessing steps included (1) compensation of systematic, slice-dependent time shifts, (2) elimination of systematic odd-even slice intensity differences caused by interleaved acquisition, and (3) rigid body correction for interframe head motion within and across runs. Each of three fMRI runs was intensity scaled to produce a whole-brain mode of 1000 , not counting the first four frames of each run (Ojemann et al., 1997). Atlas registration was achieved by computing affine transforms connecting the fMRI run first frame (averaged over all runs after cross-run alignment) with the preoperative T1and T2-weighted averages. The data were realigned to the Washington University Neuroimaging Laboratories representative adult template based on MP-RAGE data from 12 normal individuals and was made to conform to the Talairach atlas (Talairach and Tournoux, 1988) as defined by the spatial normalization procedure (Lancaster et al., 1995). Comparability of children and adult functional data in a common stereotactic space has been demonstrated previously (Burgund et al., 2002; Kang et al., 2003). The BOLD data were transformed to atlas space and resampled to $3 \mathrm{~mm}$ cubic voxels before functional connectivity analysis.

Correlation techniques. Data were temporarily low-pass filtered $(f<0.1 \mathrm{~Hz})$ and spatially smoothed $(6 \mathrm{~mm}$ full-width at halfmaximum Gaussian blur). Sources of spurious variance along with their temporal derivatives were removed from the data through linear regression: (1) six parameters obtained by rigid body correction of head motion; (2) the whole-brain signal, averaged over a fixed region in atlas space; (3) signal from a ventricular region of interest; (4) signal from a region centered in the white matter. Correlation maps were produced by extracting the BOLD time course from a seed region, then computing the correlation coefficient between that time course and the time course from all other brain voxels.

Seed regions were 12-mm-diameter spheres centered on previously published foci (Table 1). Dorsal attention system network regions included the intraparietal sulcus (IPS), frontal eye fields (FEFs), and middle temporal region (MT+) (Fox et al., 2005). Default/task-negative network seeds comprised the posterior cingulate/precuneus, medial prefrontal cortex, and lateral parietal (LP) regions (Fox et al., 2005). A memory network seed was centered on the hippocampal formation (HF) (Vincent et al., 2006) and smaller $6 \mathrm{~mm}$ diameter seeds were placed at 3 $\mathrm{mm}$ increments both anterior and posterior to this central seed. A primary auditory cortex seed was placed in Brodmann area 41 (Desai et al., 2005), and a somatomotor (SM) seed was centered in the hand region of the precentral gyrus (Fox et al., 2006a). Finally, the primary visual network (V1) and amygdala were analyzed with seeds anatomically defined on the atlas by the calcarine sulcus and amygdala, respectively.

The correlation maps were converted to normal distribution by Fischer's $z$ transform (Jenkins and Watts, 1968). These values were converted to $z$ scores by dividing the square root of the variance, calculated as $1 / \sqrt{(n-3)}$, where $n$ is the degrees of freedom of the measurement. The degrees of freedom was calculated according to Bartlett's theory (Jenkins and Watts, 1968) using the BOLD time series-lagged autocovariance function averaged over multiple regions of interest (ROIs) in both the preoperative and postoperative datasets. The correction factor for independent frames was calculated to be 2.70 . Thus, for a dataset of 588 frames (three runs of 200 frames/run, less premagnetization steady-state frames), the degrees of freedom was 588/2.70 $=217.8$. Finally, $z$ score maps were thresholded at a multiple samples corrected significance level of $p<0.001$ ( $|z|>3.3$; cluster size, 21 voxels) (McAvoy et al., 2001).

Surface-based mapping. The volumetric statistical results were projected onto the cortical surface of the PALS (population-average landmark- and surface-based) atlas by using a multifiducial mapping method that avoids the biases of choosing a single hemisphere from a single individual as an atlas target (Van Essen, 2005).

\section{Results}

We began our analysis by using manually defined ROIs that included most of the cortical gray matter of the left and right cerebral hemispheres. ROIs of the left and right thalamus were manually traced. Correlations were computed using the regional time series acquired before and after surgery (Table 2).

As shown in Table 2, correlations between left and right cortex significantly decreased after transection of the corpus callosum. This represents an $88.5 \%$ decrease in shared variance [(preoperative $r^{2}$ - postoperative $\left.r^{2}\right) /$ preoperative $r^{2}$ ] attributable to interhemispheric communication via the corpus callosum. The remaining shared variance (11.5\%) likely represents a combination of non-neuronal noise (i.e., cardiac and respiratory) (Birn et al., 2006; Shmueli et al., 2007) and synchrony arising from connections via the anterior and posterior commissures and the brainstem (Jones, 2007). Given that our data were subjected to regression of signal from white matter and ventricles before analysis, the percentage of remaining variance of non-neuronal origin likely is very small.

Callosotomy had a much smaller effect, albeit significant, on the shared variance between the two thalami (Table 2), which decreased 54\%. Not surprisingly, coherence between the cortex and ipsilateral thalamus was least affected, followed by cortex and contralateral thalamus, suggesting a contribution from callosal 

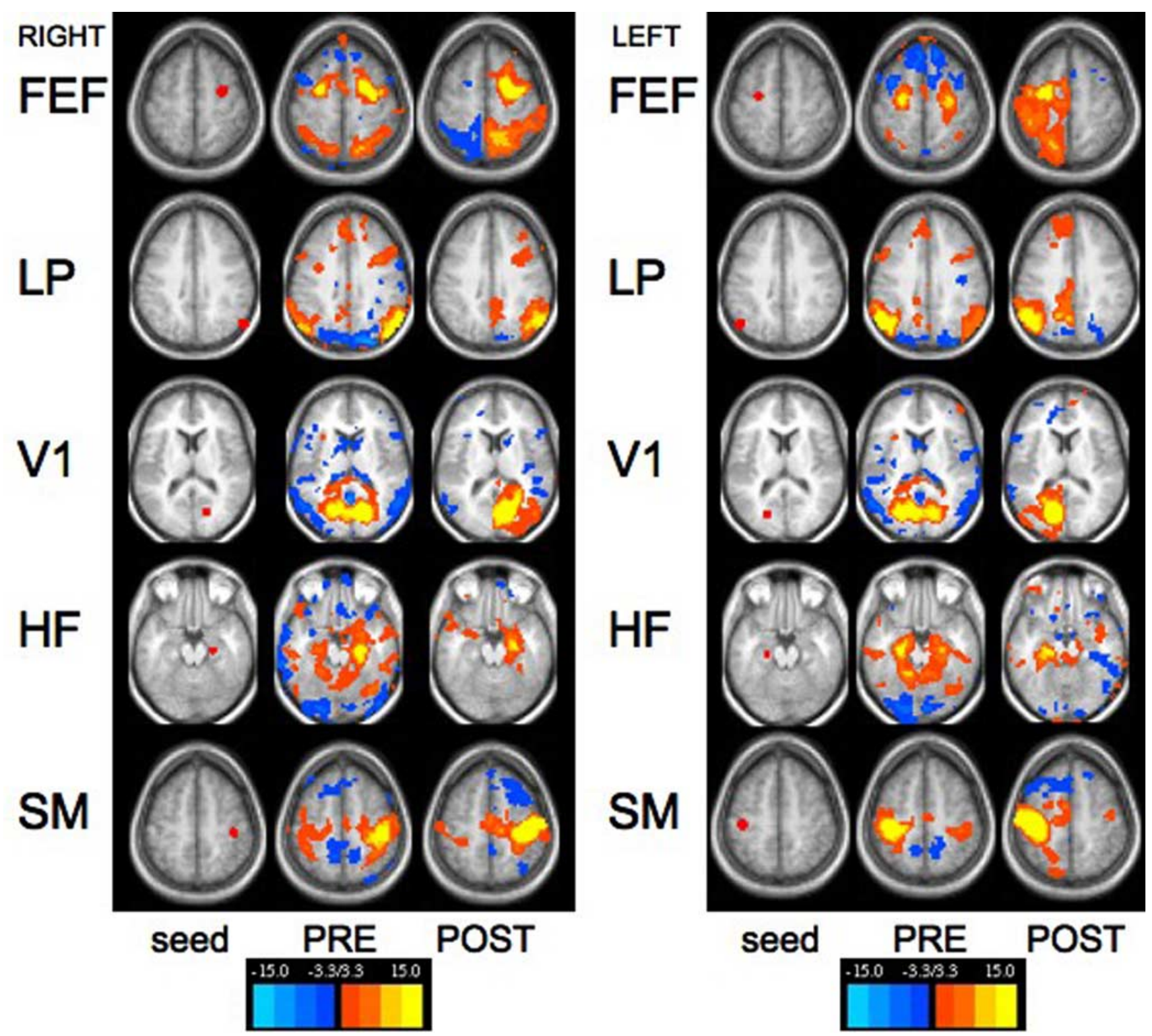

Figure 1. Functional connectivity computed before and after corpus callosotomy for right- and left-sided seed regions. Seed regions defined in the right hemisphere (first column of each set) were used to generate correlation maps both before (second column) and after (third column) complete transection of the corpus callosum. Seed ROls were placed laterally in the hemispheres to avoid confusion that might arise because of partial volume averaging associated with ROls close to the midline. Presurgery, functional connectivity maps using the selected seed regions revealed a pattern of correlations that is consistent with published literature, including near symmetric correlations with the contralateral hemisphere. For example, a seed in right lateral parietal region demonstrates correlations with the left lateral parietal as well as with medial prefrontal cortex and posterior cingulate/precuneus (second row). Postsurgery, contralateral correlations that were present before transection prominently disappear (FEF, top row; LP, second row; V1, third row). Correlations with the hippocampal formation seed and the somatomotor seed do not show complete loss of contralateral correlations. These regions have neuroanatomical connections with the opposing hemisphere through fibers that do not cross in the corpus callosum. The temporal lobe, for example, is able to communicate with the contralateral hemisphere through the anterior commissure (Schmahmann and Pandya, 2006). Findings were similar for both right- and left-sided seeds. FEF, $z=$ $55.5 ; \mathrm{LP}$, seed, $z=36.5 ; \mathrm{V1}, \mathrm{z}=12.5 ; \mathrm{HF}, \mathrm{z}=-13.5 ; \mathrm{SM}, \mathrm{z}=51.5$.

fibers to a portion of the shared variance between the cerebral cortex of one hemisphere and the contralateral thalamus.

We further analyzed our data using paired, predefined 12mm-diameter spherical seed regions corresponding to previously described nodes within the dorsal attention network (Fox et al., 2005), default network (Fox et al., 2005), somatomotor network (Fox et al., 2006a), primary auditory cortex (Desai et al., 2005), and hippocampal formation/mesial temporal lobe (Vincent et al., 2006), as well as anatomically defined seeds for the visual system (V1) and amygdala. Figure 1 shows representative $z$ score correlation maps of multiple seed regions projected onto transverse MRI slices. These seed regions correspond to the previously de- scribed dorsal attention (FEF), default (LP), primary visual (V1), memory (HF), and SM systems. All systems show a striking loss of interhemispheric functional connectivity after callosotomy with the exception of the SM and memory systems (HF). This was true for both right and left sided seeds.

Further investigation of differential temporal connectivities with seeds in the amygdala, anterior and posterior hippocampus, primary auditory cortex, and MT+ showed a progressive loss of interhemispheric correlations from rostral to caudal temporal lobe after callosotomy. Analysis of correlation maps generated from multiple seeds through the hippocampus suggest that the majority of postcallosotomy connectivity seen in the contralat- 
eral anterior temporal lobe may represent secondary amygdala-amygdala connectivity mediated via the anterior commissure (supplemental Fig. 1, available at www. jneurosci.org as supplemental material).

Seed ROIs placed in FEFs have been shown to reveal patterns of coherence within the dorsal attention system (Fox et al., 2006b) and do so in our patient as well with preservation of ipsilateral FEF/IPS functional connectivity (Fig. 1). With the exception of the somatomotor system, the roles of the thalamus, basal ganglia and diffuse projecting systems emanating from the brainstem appear limited in the maintenance of interhemispheric coherence.

Figure 2 shows $z$ score maps of a right FEF seed projected onto a smoothed cortical surface (Van Essen, 2005), both before (Fig. 2A) and after (Fig. 2B) callosotomy. Again, interhemispheric connectivity was significantly disrupted after callosotomy, whereas intrahemispheric dorsal attention system (FEF, IPS, MT+) connectivity was maintained.

\section{Discussion}

Complete section of the corpus callosum disrupts a massive component of interhemispheric anatomical connectivity. In the present case, we had the unique opportunity to obtain resting state fMRI data before and after this therapeutic intervention. This study is important because of the opportunity it offers to examine the relationship between the connectional anatomy of the brain and its large scale functional organization emerging from patterns of coherence in the spontaneous fluctuations of the fMRI BOLD signal.

Although patterns of coherence in the fMRI BOLD signal characteristically show orderly relationships among areas consistent with known connectional anatomy, details regarding the role of anatomical connectivity are sparse. In the only study to date that directly addresses this question, Vincent et al. (2007) demonstrated that the correlation structure in the anesthetized monkey oculomotor system obtained from the fMRI BOLD signal corresponded exceptionally well with retrograde tracer studies of the connectional anatomy of that system. They further went on to show that patterns of coherence between peripheral and foveal V1 in the two hemispheres, areas of the brain of the monkey that have no direct connections, were likely sustained by polysynaptic pathways that respected visual retinotopy along the eccentricity axis. A clear test of the inferences to be drawn from these observations would be to interrupt the putative anatomical connections and demonstrate an effect on the functional connectivity demonstrated with fMRI BOLD imaging. The present observations serve that purpose.

Consistent with the hypothesis that the apparent functional connectivity demonstrated by fMRI BOLD imaging is related to the anatomical connectivity of the human brain, callosotomy dramatically reduced the shared variance in the BOLD signal between the two cerebral hemispheres. Over $88 \%$ of the shared variance was eliminated. We believe that this observation con- tributes significantly to mounting evidence favoring the neurobiological relevance of the spontaneous fluctuations in the fMRI BOLD.

Examining our results in more detail reveals striking loss of interhemispheric cortical coherence at the systems level with the possible exception of the somatomotor system, hippocampal formation, and the thalamus. With regard to the somatomotor system, the seed used for this study corresponds to the hand motor region. Anatomic tracer studies in multiple species have shown limited callosal connections between corresponding distal limb motor cortices. That somatomotor synchrony is less affected by callosotomy is consistent with the existence of these "callosal holes" (Myers, 1965; Pappas and Strick, 1981; Killackey et al., 1983). Conversely, persistent somatomotor correlations after callosotomy may be caused by synchronous ascending information transmitted via somatomotor thalamocortical projections.

Likewise, interhemispheric hippocampal and amygdala correlations were partially retained after callosotomy, specifically in the contralateral amygdala and anterior lateral temporal lobe. (Fig. 1, supplemental Fig. 1, available at www.jneurosci.org as supplemental material). The known connectivity of anterior and mesial portions of the right and left temporal lobes via the anterior commissure may explain this retained postcallosotomy coherence. Anterograde autoradiographic tracing in the macaque demonstrates differential connectivity of the temporal lobes from rostral to caudal. The corpus callosum receives its most significant projections from the caudal third of the temporal lobe, with 
progressively fewer fibers found rostrally. The anterior commissure receives and transmits fibers from the entire temporal lobe, but is biased toward the rostral third of the temporal isocortex, including the temporal pole, superior and inferior temporal gyri, the parahippocampal gyrus, orbitofrontal cortex, prepyriform cortex, and the amygdala (Demeter et al., 1990; Schmahmann and Pandya, 2006). In addition, studies in both animals and humans have demonstrated the clear role of the amygdala in affective memory modulation (McGaugh et al., 1996; Cahill and McGaugh, 1998; Hamann et al., 1999) via reciprocal projections to the ipsilateral anterior hippocampus (Amaral et al., 1992; Pitkanen et al., 2000) and caudate nucleus (Packard and Wingard, 2004).

More recent work using resting functional connectivity MRI in humans has demonstrated distinct pathways for the head (anterior lateral temporal cortex, entorhinal/perirhinal cortex) and body (inferior parietal lobule, retrosplenial cortex, posterior cingulate, and ventral medial prefrontal cortex) of the hippocampus (Kahn et al., 2008). Residual interhemispheric coherence in the amygdalae and anterior lateral temporal lobes seen after callosotomy in both the anterior hippocampus and hippocampal body correlation maps (supplemental Fig. 1, available at www. jneurosci.org as supplemental material) suggests that the anterior commissure may be an important mediator of interhemispheric transfer for the more rostral pathway.

Coherence between the left and right thalamus decreased just $>50 \%$, suggesting that bilateral synchrony within the thalamus represents a contribution from callosal fibers to a portion of the shared variance between the cerebral cortex of one hemisphere and the contralateral thalamus plus a contribution likely emanating from afferent structures such as the brainstem reticular formation (Jones, 2007).

Intrahemispheric patterns of functional connectivity remained after callosotomy in all systems. This observation is consistent with a large body of neuropsychological literature documenting independent functioning of each hemisphere in callosotomy patients (Devinsky and Laff, 2003; Gazzaniga, 2005). Our data are insufficient to assess the impact of a loss of interhemispheric coherence on a system such as the dorsal attention network (Fig. 2). However, future work combining imaging of the type presented here with detailed neuropsychological evaluation preoperatively and postoperatively could be most revealing in this regard.

Consistent with other published data (Kiviniemi et al., 2000; Vincent et al., 2007), the results of this investigation also demonstrate the presence of resting state functional connectivity in a child under general propofol anesthesia. This finding is of interest for two reasons. First, the persistence of coherent network synchrony suggests that resting state BOLD modulation represents a fundamental level of brain organization that transcends levels of arousal. Second, this observation suggests a possible future utility for resting functional connectivity analysis in younger children with surgically treatable epilepsy, specifically preoperative mapping of functional systems and postoperative evaluation where general anesthesia is required.

The patient's young age, developmental delay, and history of epilepsy represent limitations of the present dataset. In addition, the data reported here may reflect an acute postoperative disconnection phenomenon and do not exclude the possibility of longterm reorganization and reconstitution of interhemispheric functional connectivity. Nonetheless, the clear loss of interhemispheric correlation with preservation of intrahemispheric networks is consistent with the view that the corpus callosum plays a major role in maintaining interhemispheric coherence of spontaneous BOLD fluctuations. These data provide unique information concerning the role of anatomic connectivity in the genesis of BOLD signal functional connectivity.

Recent publications have posited a significant role for nonneuronal factors (i.e., cardiac, respiratory, end tidal $\mathrm{CO}_{2}$ variations) in the genesis of BOLD signal correlations (Wise et al., 2004; Birn et al., 2006; Shmueli et al., 2007). Our data suggest that this contribution is minor. In the present study the upper bound for such a contribution would be $\sim 11 \%$. However, given that signals from ventricles and white matter were removed from our data before analysis, it is likely that this contribution is even less.

Future work must seek to identify and implement strategies that selectively eliminate cardiac and respiratory contributions to the spontaneous fluctuations in the fMRI BOLD signal. In doing so, we must be sensitive to the possibility that slow variations in cardiac and respiratory parameters might well have their origin within the brain. Thus, just as spontaneous variations in the fMRI BOLD signal contribute to variability in human behavior (Fox et al., 2007), so also might variability in brain systems regulating cardiac and respiratory functions lead to variability in the functions themselves. This is a researchable question that deserves investigation.

Finally, we note that the data presented in this article are publicly available at http://www.brainscape.org/along with software tools useful in their analysis.

\section{References}

Amaral DG, Price JL, Pitkanen A, Carmichael ST (1992) Anatomical organization of the primate amygdaloid complex. New York: Wiley.

Berger H (1929) Über das Elektroenkephalogramm des Menschen. Archiv für Psychiatrie und Nervenkrankheiten 87:527-570.

Birn RM, Diamond JB, Smith MA, Bandettini PA (2006) Separating respiratory-variation-related fluctuations from neuronal-activity-related fluctuations in fMRI. NeuroImage 31:1536-1548.

Biswal B, Yetkin FZ, Haughton VM, Hyde JS (1995) Functional connectivity in the motor cortex of resting human brain using echo-planar MRI. Magn Reson Med 34:537-541.

Burgund ED, Kang HC, Kelly JE, Buckner RL, Snyder AZ, Petersen SE, Schlaggar BL (2002) The feasibility of a common stereotactic space for children and adults in fMRI studies of development. NeuroImage 17:184-200.

Cahill L, McGaugh JL (1998) Mechanisms of emotional arousal and lasting declarative memory. Trends Neurosci 21:294-299.

Demeter S, Rosene DL, Van Hoesen GW (1990) Fields of origin and pathways of the interhemispheric commissures in the temporal lobe of macaques. J Comp Neurol 302:29-53.

Desai R, Liebenthal E, Possing ET, Waldron E, Binder JR (2005) Volumetric vs. surface-based alignment for localization of auditory cortex activation. NeuroImage 26:1019-1029.

Devinsky O, Laff R (2003) Callosal lesions and behavior: history and modern concepts. Epilepsy Behav 4:607-617.

Fox MD, Raichle ME (2007) Spontaneous fluctuations in brain activity observed with functional magnetic resonance imaging. Nat Rev Neurosci 8:700-711.

Fox MD, Snyder AZ, Vincent JL, Corbetta M, Van Essen DC, Raichle ME (2005) The human brain is intrinsically organized into dynamic, anticorrelated functional networks. Proc Natl Acad Sci USA 102:9673-9678.

Fox MD, Snyder AZ, Zacks JM, Raichle ME (2006a) Coherent spontaneous activity accounts for trial-to-trial variability in human evoked brain responses. Nat Neurosci 9:23-25.

Fox MD, Corbetta M, Snyder AZ, Vincent JL, Raichle ME (2006b) Spontaneous neuronal activity distinguishes human dorsal and ventral attention systems. Proc Natl Acad Sci USA 103:10046-10051.

Fox MD, Snyder AZ, Vincent JL, Raichle ME (2007) Intrinsic fluctuations within cortical systems account for intertrial variability in human behavior. Neuron 56:171-184.

Gazzaniga MS (2005) Forty-five years of split-brain research and still going strong. Nat Rev Neurosci 6:653-659. 
Hamann SB, Ely TD, Grafton ST, Kilts CD (1999) Amygdala activity related to enhanced memory for pleasant and aversive stimuli. Nat Neurosci 2:289-293.

Jenkins GM, Watts DG (1968) Spectral analysis and its applications. San Francisco: Holden-Day.

Jones EG (2007) The thalamus. Cambridge, UK: Cambridge UP.

Kahn I, Andrews-Hanna JR, Vincent JL, Snyder AZ, Buckner RL (2008) Distinct cortical anatomy linked to subregions of the medial temporal lobe revealed by intrinsic functional connectivity. J Neurophysiol, in press.

Kang HC, Burgund ED, Lugar HM, Petersen SE, Schlaggar BL (2003) Comparison of functional activation foci in children and adults using a common stereotactic space. NeuroImage 19:16-28.

Killackey HP, Gould III HJ, Cusick CG, Pons TP, Kaas JH (1983) The relation of corpus callosum connections to architectonic fields and body surface maps in sensorimotor cortex of new and old world monkeys. J Comp Neurol 219:384-419.

Kiviniemi V, Jauhiainen J, Tervonen O, Paakko E, Oikarinen J, Vainionpaa V, Rantala H, Biswal B (2000) Slow vasomotor fluctuation in fMRI of anesthetized child brain. Magn Reson Med 44:373-378.

Lancaster JL, Glass TG, Lankipalli BR, Downs H, Mayberg H, Fox PT (1995) A modality-independent approach to spatial normalization of tomographic images of the human brain. Hum Brain Mapp 3:209-223.

McAvoy MP, Ollinger JM, Buckner RL (2001) Cluster size thresholds for assessment of significant activations in fMRI. NeuroImage 13 [Suppl]:S198.

McGaugh JL, Cahill L, Roozendaal B (1996) Involvement of the amygdala in memory storage: interaction with other brain systems. Proc Natl Acad Sci USA 93:13508-13514.

Myers RE (1965) Functions of the corpus callosum. Boston: Little, Brown.

Ojemann JG, Akbudak E, Snyder AZ, McKinstry RC, Raichle ME, Conturo TE (1997) Anatomic localization and quantitative analysis of gradient refocused echo-planar fMRI susceptibility artifacts. NeuroImage 6:156-167.

Packard MG, Wingard JC (2004) Amygdala and "emotional” modulation of the relative use of multiple memory systems. Neurobiol Learn Mem 82:243-252.

Pappas CL, Strick PL (1981) Anatomical demonstration of multiple representation in the forelimb region of the cat motor cortex. J Comp Neurol 200:491-500.

Pitkanen A, Pikkarainen M, Nurminen N, Ylinen A (2000) Reciprocal connections between the amygdala and the hippocampal formation, perirhinal cortex, and postrhinal cortex in rat. A review. Ann NY Acad Sci 911:369-391.

Posner MI, Raichle ME (1998) The neuroimaging of human brain function. Proc Natl Acad Sci USA 95:763-764.

Schmahmann JD, Pandya DN (2006) Fiber pathways of the brain. Oxford: Oxford UP.

Shmueli K, van Gelderen P, de Zwart JA, Horovitz SG, Fukunaga M, Jansma JM, Duyn JH (2007) Low-frequency fluctuations in the cardiac rate as a source of variance in the resting-state fMRI BOLD signal. NeuroImage 38:306-320.

Talairach J, Tournoux P (1988) Co-planar stereotaxic atlas of the human brain. New York: Thieme Medical.

Van Essen DC (2005) A population-average, landmark- and surface-based (PALS) atlas of human cerebral cortex. NeuroImage 28:635-662.

Vincent JL, Snyder AZ, Fox MD, Shannon BJ, Andrews JR, Raichle ME, Buckner RL (2006) Coherent spontaneous activity identifies a hippocampal-parietal memory network. J Neurophysiol 96:3517-3531.

Vincent JL, Patel GH, Fox MD, Snyder AZ, Baker JT, Van Essen DC, Zempel JM, Snyder LH, Corbetta M, Raichle ME (2007) Intrinsic functional architecture in the anaesthetized monkey brain. Nature 447:83-86.

Wise RG, Ide K, Poulin MJ, Tracey I (2004) Resting fluctuations in arterial carbon dioxide induce significant low frequency variations in BOLD signal. NeuroImage 21:1652-1664. 